\title{
El tratamiento con ibuprofeno en niños con sinovitis transitoria de cadera redujo la duración de los síntomas
}

A randomized clinical trial:Should the child with transient synovitis of the hip be treated with nonsteroidal anti-inflammatory drugs? Kermond S, Fink M, Graham K y col.Ann Emerg Med. 2002;40:294-299.

\section{Objetivo}

Evaluar el efecto del tratamiento con ibuprofeno $(10 \mathrm{mg} / \mathrm{kg} / \mathrm{dia}$ en tres tomas por 5 días) sobre la duración de los síntomas de la sinovitis transitoria de cadera (STC).

\section{Diseño}

Ensayo clínico aleatorizado doble ciego controlado con placebo. Los síntomas fueron monitorizados por un diario seguido por los padres y por control médico.Se calculó un tamaño muestral de 17 pacientes por grupo para tener un poder del $80 \%$ para detectar una diferencia de 2 días en la duración de los síntomas, asumiendo que la sintomatología se resolvería en un $95 \%$ entre el $1 \stackrel{0}{0}$ y el 9 día de evolución.

\section{Lugar}

Departamento de Emergencias del Hospital de Niños Real de Melbourne, Australia.

\section{Pacientes}

Niños con diagnóstico clínico de STC, en base a dolor y/o cojera, con limitación de los movimientos de la cadera o dolor localizado en esa articulación en el examen físico. Se utilizaron criterios de exclusión muy estrictos para evitar cualquier potencial enmascaramiento de patologías más severas por el efecto antiinflamatorio del ibuprofeno. Criterios de exclusión: Edad menor a 12 meses y mayor a 12 años, duración de los síntomas mayor a 1 semana, fiebre mayor a 38으 $\mathrm{C}$ medida en la guardia, recuento de leucocitos mayor a $15.000 \mathrm{cel} / \mathrm{mm} 3$, eritrosedimentación mayor a $25 \mathrm{~mm} / \mathrm{h}$, radiografía de cadera patológica, requerimiento de internación, patología previa de la cadera, contraindicación al uso de ibuprofeno.

\section{Intervención}

Se aleatorizó a los pacientes para recibir ibuprofeno $10 \mathrm{mg} / \mathrm{kg} / \mathrm{do}-$ sis en tres tomas por 5 días $(n=17)$ o placebo $(n=19)$. Los padres e investigadores eran ciegos a la intervención. Los pacientes también podían recibir paracetamol. Se les realizó una ecografía de cadera dentro de las $24 \mathrm{Hs}$ del ingreso y a los 7 días. El diario debía ser completado 2 veces por día por 7 días. Allí se debía registrar la medicación suministrada, la necesidad de uso de paracetamol, cualquier problema hallado y completar una escala de dolor para los padres y el niño y un cuestionario de síntomas. Los pacientes eran reevaluados a los 2-3 días del ingreso y después según indicación clínica.

\section{Medición de resultados principales}

La resolución de los síntomas se definió cuando el diario indicaba ausencia de dolor, marcha normal y realización de las actividades nor males.Los pacientes incluídos se analizaron según intención de tratar.

\section{Resultados Principales}

La mediana de duración de los síntomas fue de 2 días en el grupo iburofeno y de 4.5 días en el grupo placebo $(p=0.05)$.A los 7 días, había 2 pacientes en el grupo ibuprofeno y 4 en el grupo placebo aún con síntomas.Se reportaron sintomas gastrointestinales leves en 4 pacientes del grupo ibuprofeno y en 2 del grupo placebo. Una paciente (grupo ibuprofeno) continuaba con síntomas al mes y fue derivada al reumatólogo.Su ecografía inicial y al $7^{\circ}$ día mostraba engrosamiento de la sinovial sin derrame asociado.Todos los demás pacientes resolvieron sus síntomas sin otro tratamiento.

\section{Conclusiones}

El tratamiento con ibuprofeno disminuyó la duración de los síntomas de STC.

Fuente de financiamiento:The Boots Company PLC, Melbourne, Australia.

\section{Comentario}

La STC, también llamada sinovitis tóxica o cadera irritable, es una enfermedad benigna autolimitada que se presenta comúnmente en niños de 3 a 10 años 1 . Se trata de una inflamación estéril de la articulación de la cadera que se manifiesta con fiebre no muy elevada, dolor y limitación del movimiento de la cadera, rengueo y/o claudicación de la marcha'.Si bien la causa es desconocida, se atribuye a un fenómeno postinfeccioso. En general, se resuelve espontáneamente antes de los 7 días, pero genera angustia en padres y niños. A los médicos nos genera preocupación su diagnóstico diferencial con la artritis séptica de cadera, una enfermedad mucho más grave que requiere internación, punción y drenaje a cielo abier to ${ }^{2,3}$.Los autores propusieron criterios de exclusión muy estrictos para evitar esta contingencia y no tuvieron casos de reingresos por artritis séptica u osteomielitis (si bien la muestra fue muy pequeña para poder asegurar fehacientemente el diagnóstico diferencial mediante estos criterios). Es incierto cuan inclusivo se puede ser sin que resulte en confundir una STC con el inicio de una artritis séptica mal diagnosticada.Por este motivo considero importante respetar los criterios de exclusión utilizados por los autores aún sabiendo que muchos de los excluidos terminarán siendo STC (resignar algo de sensibilidad para ganar especificidad). Existen otros diagnósticos diferenciales como inicio de una enfermedad reumática, enfermedad de Perthes y epifisiolisis que suelen tener un curso subagudo o crónico y podrían ser confundidas con STC, por lo que si se decide usar AINE para la STC y no se constata mejoría, se debería discontinuar el tratamiento luego de 5 días y replantear el diagnóstico.

Conclusión del comentador: El uso de ibuprofeno acorta la duración de los sintomas en la STC.Se deben respetar los criterios de exclusión y la duración del tratamiento utilizados por los autores. Todos los niños con diagnóstico de STC deben ser estrechamente controlados clínicamente sean o no tratados con AINE.

\section{Dr. Juan Pablo Mouesca [ Pediatra ]}

\section{Referencias}

1. Staheli L. Sinovitis tóxica (cadera irritable).En: Nelson Tratado de pediatría.14 Ed. Behrman R.KliegmanR. Nelson W.Waughan V. Editores. Interamericana - Mc Graw- Hill-pg. 2073-4 2.Schwentker E.Artritis séptica. En:Hoekelman Atención primaria en pediatria. Hoekelman R.Friedman S.Nelson n.Seidel M.Waitzman M. Editores. Harcourt Brace. 1998;257:1577-9 3. Rivas N Lopez E. Artritis séptica.En Guia de diagnóstico y tratamiento.Edición 2000.Asociación de profesionales del Hospital de Niños R. Gutierrez. 